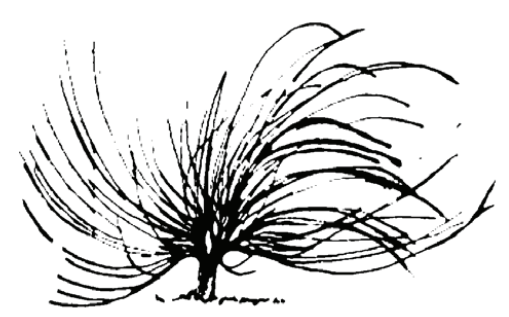

\title{
El Perfil Tecnológico de un Profesor de Idiomas en México: Una Revisión de Estándares
}

\author{
César Augusto Borromeo García ${ }^{1}$ \\ Universidad Veracruzana \\ Xalapa, Ver., México \\ cesar.bogc@gmail.com
}

\section{Resumen}

La estandarización es un proceso que se ha venido incrementando en los últimos años gracias a la globalización. La enseñanza del idioma inglés se rige por la estandarización y uniformización de procesos y conocimientos. Entre estos, se encuentran los conocimientos y procesos relacionados con las Tecnologías de la Información y la Comunicación (TIC). Sin embargo, realizar una medición en contextos con condiciones distintas a otra resulta un proceso que muchos consideran incorrecto. En este artículo, se presenta la selección de estándares internacionales en materia de TIC en la enseñanza del inglés para un contexto mexicano. Primeramente, se presentan antecedentes del contexto mexicano. Después se muestra el proceso de

Recibido: 30 de junio de 2015-Aprobado: 26 de noviembre de 2015

1 Estudiante de la Maestría en Educación Virtual (MEV) del Instituto de Investigaciones en Educación (IIE) de la Universidad Veracruzana. Licenciado en Lengua Inglesa por la Universidad Veracruzana. Profesor de Inglés en Kiosk, Escuela de Idiomas. 
comparación y la descripción de la literatura seleccionada. El artículo finaliza con una propuesta de estándares que podrían ser adecuados en el contexto mexicano.

Palabras clave: estándares, TIC, profesor, educación, lengua inglesa, México

\begin{abstract}
Standardization is a process that has been increasing in the past years because of globalization. English Language Teaching is ruled by standardization and uniformization of processes and knowledge. It is possible to find topics related to Information and Communication Technologies (ICT) among these knowledge and processes. Nevertheless, measuring different contexts with different conditions with the same considerations is a process that many consider incorrect. In this paper, a selection of international standards regarding ICT in English Language Teaching is proposed. First, background of the Mexican context is presented. Next, the process of comparison and the description of the selected literature is shown. Finally, a suggestion of adequate standards for the Mexican context is presented.
\end{abstract}

Keywords: standards, ICT, teacher, education, English language, Mexico

\title{
Introducción
}

La enseñanza de idiomas, principalmente el inglés, es una de las disciplinas en la que, desde hace muchos años se integran las Tecnologías de la Información y la Comunicación (TIC) de una manera constante. Sin embargo, esta integración se ha dado de una forma más independiente gracias a los profesores y no a los organismos. Al notar esto, las autoridades y principales impulsores de la enseñanza del inglés se han preocupado por desarrollar estándares, planes de integración de tecnología y perfiles y marcos de referencia para guiar una incorporación más fluida. Estos planes han sido pensados para contextos generales o internacionales, es decir, son susceptibles a ser modificados para contextos específicos. 
En México no existen perfiles o marcos de referencias suficientes para guiar una integración de TIC fluida para la disciplina de la enseñanza del idioma inglés. De esta forma, surge la necesidad de conocer el contexto mexicano y observar, a partir de las pocas referencias existentes, qué cambios pueden hacerse a los estándares internacionales sugeridos por las autoridades de la enseñanza de inglés para poder ser aplicados en México. En este artículo, se desarrolla una propuesta de adaptación de los principales estándares, perfiles y marcos de referencia internacionales, de manera que estén adaptados al contexto mexicano. Para esto, la propuesta se basa en la revisión de los perfiles existentes en México.

\section{Objetivos}

Este trabajo tiene como objetivo encontrar los estándares mínimos que debe tener un profesor del idioma inglés en México. Asimismo, se describirá cada estándar. Adicionalmente, se crearán pautas que guiarán la creación de una guía de entrevistas

\section{Justificación}

En México, existe una limitada cantidad de información respecto a estándares, perfiles y marcos de referencia sobre tecnología para profesores del idioma inglés. Aquellos que existen están enfocados más en el nivel de idioma inglés comprobable mediante certificaciones, pero en cuanto a uso de tecnología no mencionan mucho. Los documentos principales que hablan sobre integración de tecnología por parte de profesores de lengua inglesa son documentos internacionales. No obstante, estos documentos, al no estar dirigidos a un contexto específico, carecen de las consideraciones propias de un contexto, tales como alcances, potencialidades y limitaciones. Por ende, para lograr crear un perfil tecnológico para profesores en México es necesario considerar el contexto propio. Al hacerlo, se pueden crear pautas más enfocadas para México. De esta forma, la revisión de bibliografía presentada a continuación tiene como fin brindar una propuesta de perfil tecnológico de profesores de lengua inglesa para México. 


\section{Antecedentes}

Un estándar es una forma de medir cuál es el progreso en relación con una meta (Ravitch, 1995). Ravitch (1995) menciona que dependiendo del contexto, un estándar puede ser obligatorio (requerido por una autoridad), voluntario (abierto y disponible a quien lo desee) o de facto (aceptado como lo normal por una gran cantidad de sujetos). Los estándares en educación se pueden dividir en tres tipos: de contenido (lo que un estudiante debe recibir de su profesor en materia de temas), de desempeño (lo que un sujeto-profesor o estudiante-debe dominar sobre un cierto tema) y de oportunidad de aprender (sobre disponibilidad de recursos e infraestructura).

La internacionalización de los estándares se da en un contexto global impactado en gran forma por una globalización del conocimiento (Brünner, 2003; Castells, 1999). El manejo de empresas económicas y aquellas que se manejan como empresas económicas, cual es el caso de las instituciones de educación superior (Clark, 1983), se da un en mundo que cambia constantemente dependiendo de las necesidades globales. La especialización y la uniformización, procesos comunes en una sociedad global industrializada (Toffler, 1980), se han vuelto tan comunes en distintas disciplinas del conocimiento que se tiene la idea de que son universales; no se toma en cuenta que todos los contextos son distintos y cada uno requiere un tratamiento especial, aún cuando existen procesos de uniformización. Una de esas disciplinas afectadas altamente por estas creencias es la enseñanza de idiomas, en específico, el inglés.

Richards $(2008,2011)$ asevera que la enseñanza del idioma inglés es una especialización en el campo de la educación que requiere conocimiento especializado, y donde su entrada y permanencia se logran mediante características específicas y con base en estándares. Los estándares, empero, deben ser vistos como una guía o pautas que incentiven la creatividad, el pensamiento crítico y las expresiones individuales (Murphy-Judy y Youngs, 2006), pero que permitan comparación internacional entre sujetos con características similares, a fin de permitir su autoevaluación y crecimiento profesional (Brünner, 2003; Richards, 2011).

En el contexto mexicano, la situación no es distinta. Los profesores deben tener ciertos conocimientos que puedan ser demostrados y deben ser transmitidos a los estudiantes. Entre estos se incluyen los 
tecnológicos (Alarcón y Ortiz, 2014). Así, todos los profesores, incluyendo los del idioma inglés, deberían tener un cierto dominio de las Tecnologías de la Información y la Comunicación (TIC) para poder apoyar sus clases con ellas, siguiendo las necesidades de las instituciones donde laboren, incluyendo las necesidades tecnológicas (Richards y Farrell, 2005).

Diversos autores (Carney y Foss, 2008; Garrett, 2009; Gruba y Hinkelman, 2012; Harmer, 2001; Piper, 1987; Ramírez-Martinell, Casillas y Contreras, 2014; Salsbury y Crummer, 2008) aseveran que la tecnología va de la mano con la enseñanza de idiomas. Esto demuestra que es innegable que diversas TIC son necesarias en la enseñanza y aprendizaje de idiomas. Sin embargo, la tecnología sigue siendo evadida en las clases por los profesores, quizá porque carecen de los conocimientos necesarios (Alarcón y Ortiz, 2014; Crovi, 2009; Rodarte, 2014), porque su implementación significa afrontar retos, crear planes de clase exclusivos para su incursión (O’Donoghue, 2014), por cuestiones de género (Wells, 2010) o por no considerarla un recurso que solucione problemas en la enseñanza de idiomas (Chapelle, 2001). Sea cual sea la razón, se debe pensar como importante que el profesor cuente con estos conocimientos de manejo de TIC, incluyendo en México, y sea así el profesor el que decida si implementar las TIC en su aula, y no agentes externos como políticas institucionales o editoriales de libros de texto e incluso presión social, de pares o de estudiantes.

Para el contexto mexicano de educación del idioma inglés, existen documentos de la Secretaría de Educación Pública (SEP) a través del Programa Nacional de Inglés en la Educación Básica (PNIEB) (SEP, 2011a, 2011b) que hacen referencia a profesores de lengua inglesa para un nivel educativo básico (de preescolar a secundaria). Sin embargo, el mismo perfil que proponen no contiene una lista de características necesarias para el profesor de lengua inglesa. Por otro lado, un documento de la SEP (s.f.) muestra que el perfil del docente de la asignatura de inglés debe tener un nivel de inglés B2 mínimo. El mismo documento menciona que este nivel puede ser comprobable mediante una certificación internacional, ya sea $\mathrm{FCE}^{2}$ de Cambridge, IELTS $^{3}$ o TOEFL (iTP

2 FCE: First Certificate in English, un certificado elaborado por Cambridge para medir el nivel de inglés.

3 IELTS: International English Language Testing System, una certificación para medir el nivel de inglés. 
o iBT $)^{4}$. El perfil del docente de inglés de la SEP se limita únicamente a eso, al dominio del inglés comprobable mediante una certificación. Es imposible, sin embargo, encontrar referencias sobre los conocimientos de manejo de TIC que el profesor mexicano de lengua inglesa debe tener.

De esta manera, se decidió que era necesario buscar documentos que fueran estándares, marcos de referencia o perfiles deseables que mostraran las características necesarias de un profesor del idioma inglés. Preferentemente, se buscaron documentos de referencia que encajaran en un contexto mexicano o que fueran de instancias profesionales, que tengan una importancia de peso en México.

\section{Metodología}

\section{Consideraciones previas a la comparación}

El presente trabajo se desglosa de la tesis de maestría La relación entre la apropiación tecnológica de los profesores de la Licenciatura en Lengua Inglesa de la Universidad Veracruzana y la implementación de las TIC en su aula. Como parte de la recolección de datos, se debía preparar una guía de entrevista. Sin embargo, para saber qué preguntar, primero se debió revisar lo que existía sobre el tema de estándares tecnológicos de profesores de inglés. Entonces, se realizó una revisión de estándares tecnológicos de profesores de inglés para obtener una guía de entrevista. El resultado son 6 estándares básicos que a futuro se convertirían en la base para la guía de entrevistas.

La guía de entrevista es una parte de la entrevista que se sugiere se use para evitar perder el hilo conductor durante una entrevista (Ander-Egg, 1995; Garza, 2004; Gordo y Serrano, 2008; Keats, 1988). Ésta deberá ser creada a partir de la correcta operacionalización de las variables (Gordo y Serrano, 2008). Es considerada la herramienta más importante del entrevistador, y su construcción merece todos los cuidados necesarios posibles (Keats, 1992). Por ende, la revisión de los estándares tecnológicos de los profesores de inglés forma parte esencial de la construcción de dicha guía.

4 TOEFL (iTP/iBT): Test of English as a Foreign Language (Institutional Testing Program/ Internet Based Test), una certificación para medir el nivel de inglés, se presenta en diversos formatos, en este caso institucional (iTP) o mediante Internet (iBT). 


\section{Búsqueda y selección de material}

Para lograr la selección, primero fue necesario buscar los documentos de referencia. Estos debían ser estándares, marcos o perfiles deseables tecnológicos para profesores de inglés elaborados por instancias educativas mexicanas de renombre o instancias internacionales reconocidas en la disciplina y que tuvieran un alto impacto en la educación de lengua inglesa en México.

Luego se hizo una lectura de las referencias y se acomodaron en tablas. Después se compararon las referencias para observar coincidencias y diferencias. Finalmente, se organizó una tabla con estándares aceptados y para lograr un mejor manejo de la información obtenida se cambió el nombre del estándar a uno más corto y que reflejara el tema del que se habla (ver Tabla 1 para los nombres). A continuación, se detallan los pasos a seguir, el contenido de los documentos comparados y el resultado de la comparación.

Se inició una búsqueda de estándares, marcos o perfiles deseables para profesores de inglés realizados por instancias oficiales. La búsqueda se llevó a cabo en repositorios digitales, instancias gubernamentales y organizaciones internacionales de formación en lengua inglesa. La directriz de esta búsqueda fue la de encontrar documentos que contuvieran características sobre el conocimiento tecnológico, pero que fueran hechos por instancias certificadoras o de alta importancia en la enseñanza del inglés.

Se obtuvieron resultados de estándares, marcos y perfiles del British Council (para la India y Reino Unido), Educational Testing Service (ETS, Estados Unidos de América), Michigan State (Estados Unidos de América), CEA Standards for English Language Programs and Institutions (Estados Unidos de América), STELLA Standards Framework (Australia), Teachers'Standards del Departamento de Educación británico (Reino Unido), Consejo Nacional para la Calidad de la Educación Turística (CONAET, México), de la Secretaría de Educación Pública (SEP, México), Center for Applied Linguistics (CAL, Estados Unidos de América), English Language Teacher Guidelines (Gobierno de Irlanda), la Universidad de Cambridge (Reino Unido), el National Council of Teachers of English (NCTE, Estados Unidos de América), la asociación Teachers of English of Students of Other Languages (TESOL, Estados Unidos de América) y la Universidad de Southampton 
(Reino Unido). Los documentos, entonces, describen en su mayoría a un profesor en un contexto distinto al mexicano.

La mayoría de estos documentos describen las directrices que debe seguir el profesor de inglés como un todo, no sólo en el ámbito tecnológico. Es decir, describen todos los aspectos deseables de un profesor integral de lengua inglesa. Al tratar temas tan amplios, el uso de la tecnología es apenas mencionado en los últimos 4 de la lista anterior (Universidad de Cambridge, National Council of Teachers of English, Teachers of English of Students of Other Languages y la Universidad de Southampton) los que describen más ampliamente el aspecto tecnológico de un profesor de inglés.

Se decidió entonces que los principales estándares a ser usados serían los de TESOL (2008), Cambridge (2014a, 2014b, 2014c), NCTE (1996) y la Universidad de Southampton (s.f.). Estos fueron los más completos y apropiados para el contexto mexicano debido a que se encuentran más adentrados a las certificaciones necesarias mencionadas en el perfil de la SEP (SEP, s.f.), el único marco que se pudo encontrar para profesores de lengua inglesa en México. Todos estos documentos incluyen un perfil deseable o estándares deseables de un profesor o de un estudiante del idioma inglés. Todos, además, incluyen la tecnología como una parte de la formación docente de un profesor de inglgicamente hablando puede estar desactualizado, la falcnologo de un estudiante del idioma ingls de la SEP del PNIEB que hacen refés. Sin embargo, únicamente TESOL incluye un documento en extenso sobre este tema de tecnología, es decir, sólo TESOL tiene un documento exclusivamente enfocado en los estándares tecnológicos de un profesor de lengua inglesa.

Se llegó así al TESOL Technology Standards Framework (2008). Este sería la base con el que se compararían los demás. Esta decisión se basa en varias premisas. La primera es que a pesar de ser un documento que tecnológicamente hablando puede estar desactualizado (debido a la rapidez de los cambios tecnológicos), la decisión de evitar nombrar tecnología específica permite que el documento mantenga su vigencia (TESOL, 2008). En vez de eso, el documento nombra habilidades, disposiciones y características tecnológicas de un profesor de inglés.

La segunda razón radica en la importancia que tiene TESOL como organización alrededor del mundo y su reconocimiento como autoridad en la enseñanza del inglés como segunda lengua o lengua extranjera. Richards $(2008,2011)$ asevera que TESOL, sus métodos, 
recomendaciones y publicaciones tienen un impacto importante en la enseñanza del idioma.

La tercera razón es que el documento de TESOL es el más completo en cuanto a tecnología. No se encontró un documento de una instancia oficial que hablara exclusivamente de tecnología para el profesor de inglés. Aunque los documentos principales sí hacen referencia al uso de la tecnología, ninguno ahonda en ellos como lo hace TESOL. Asimismo, TESOL incluye los indicadores para cada estándar.

\section{Descripción de los documentos revisados}

\section{TESOL - Technology Standards Framework, 2008}

La asociación TESOL (Teachers of English of Students of Other Languages, o Asociación de Profesores de Inglés para Estudiantes de Otros Idiomas, por sus siglas en inglés), creó un documento exclusivamente basándose en el perfil tecnológico deseado de un profesor y un estudiante del idioma inglés.

El extenso, TESOL Technology Standards Framework, fue desarrollado pensando en brindar directrices tecnológicas para estudiantes y profesores del idioma (TESOL, 2008). Aunque el documento cuenta con estándares para estudiantes y estándares para profesores, sólo se analizaron los estándares referentes al profesor de inglés. Por ende, esta revisión no incluye un análisis de los estándares de estudiantes de acuerdo con TESOL.

Los estándares del profesor son 14. Cada uno cuenta con sus indicadores de desempeño. Adicionalmente, el concepto completo de cada estándar fue tomado de Healey y Hubbard (s.f.), quienes también son los autores del documento de TESOL.

\section{Cambridge University - Cambridge English Teaching Fra- mework, 2014}

La Universidad de Cambridge creó el marco de referencia para la enseñanza del inglés. El documento sirve para apoyar a los profesores a identificarse en dónde están en su carrera profesional y dar pautas y directrices para desarrollarse profesionalmente en aquellas áreas de oportunidad (Cambridge English, 2014a, 2014b, 2014c). 
El marco completo consta de cinco documentos, pero sólo los últimos tres son los que contienen la información completa referente a su teoría, los primeros dos son documentos únicamente introductorios. Fueron, entonces, los últimos tres los que se tomaron para la comparación de estándares.

Estos documentos, sin embargo, contienen información sobre el perfil completo de un profesor. Es decir, no se especializan en un perfil tecnológico. No obstante, sí existe una referencia al uso de la tecnología. Esta es la principal razón por la cual no se tomó el documento como la base y, en vez de eso, fue comparado.

Nacional Council of Teachers of English NCTE - Standards for the English Language Arts, 1996

El documento es el resultado de una necesidad de tener directrices en los Estados Unidos sobre lo que debe saber un estudiante del idioma inglés. Contiene doce estándares, los cuales están enfocados a estudiantes. Son el mínimo en el dominio y habilidades relacionadas con el idioma inglés que un estudiante debería tener. Por esta razón, el documento no hace referencia a estándares de los profesores. Así, se decidió que se tomarían los estándares como el mínimo que un profesor de lengua inglesa debería saber para poder enseñarlo efectivamente a los estudiantes.

De los doce estándares, uno se enfoca en tecnología. El estándar número 8 es el estándar que ocupa la presente comparación. Dicta que "los estudiantes usan una variedad de recursos informacionales y tecnológicos (por ejemplo, librerías, bases de datos, redes computacionales, videos) para reunir y sintetizar información y para crear y comunicar el conocimiento" (NCTE, 1996, p. 28). Para lograr esto, la institución escolar debe permitir el acceso a la tecnología tanto a estudiantes como a profesores, a fin de que el aprendizaje se dé en ambas partes, volviéndose el profesor en ocasiones un aprendiz de la tecnología (NCTE, 1996).

University of Southampton - European Profile for Language Teacher Education. A Frame of Reference, s.f.

Este documento, un folleto desarrollado por la Universidad de Southampton, Reino Unido, fue creado con la finalidad de brindar a los actuales y futuros profesores de idiomas (no sólo de inglés) un número 
de estrategias, habilidades y conocimientos que mejoren sus prácticas de enseñanza de idiomas (Universidad de Southampton, s.f.).

El marco de referencia es una colección de 40 ítems, divididos en cuatro secciones: 1) estructura, 2) conocimiento y entendimiento, 3 ) estrategias y habilidades, y 4) valores. De estos, los que interesan en la comparación serán la segunda sección: conocimiento y entendimiento. En ella se encuentran dos referencias tecnológicas (la 17 y la 18) que el profesor de idiomas debe saber: (17.) entrenamiento en TIC para usos pedagógicos en el salón de clase y (18.) entrenamiento en TIC para la planeación personal, organización y descubrimiento de recursos.

\section{La comparación}

Los documentos seleccionados se compararon entonces con los estándares tecnológicos de TESOL. La comparación se llevó a cabo creando una tabla de cuatro columnas, una para cada referencia comparada (TESOL, Cambridge, NCTE, y Southampton). Únicamente los estándares de TESOL fueron descritos en su totalidad en las filas de la columna de TESOL (18 filas, de las cuales 4 fueron de categorías de TESOL y 14 de estándares), mientras que las columnas restantes llevaron una paloma de checado si su documento coincidía con algún estándar de TESOL.

Se encontró que sólo dos estándares de TESOL coincidían con los estándares, perfiles o marcos de los demás en su totalidad. Las coincidencias en TESOL fueron: 1.2. los profesores de idioma demuestran un conocimiento de una amplia variedad de apoyos tecnológicos para la enseñanza de idiomas y opciones para usarlas en un contexto definido (TESOL, 2008, p. 29), y 2.3. los profesores de idioma diseñan y administran actividades y tareas de aprendizaje de idioma de manera apropiada para lograr las metas y objetivos curriculares (TESOL, 2008, p. 34).

Se decidió que estos coincidentes fueran 'los mínimos definitivos'. Sin embargo, al realizar el análisis se detectó que el primer estándar de TESOL es la base para los demás. Este dicta: los profesores de idiomas demuestran conocimiento y habilidades en conceptos tecnológicos y competencia operacional básica, así como capacidad para igualar o incrementar los conocimientos tecnológicos de sus estudiantes en cualquier situación en que enseñen (TESOL, 2008, p. 29) Por esta razón, fue incluido dentro de los 'mínimos definitivos', aún cuando un documento (Universidad de Southampton, s.f.) no coincidía con TESOL. 
En un principio, estos 'mínimos definitivos' serían los únicos estándares que se tomarían en cuenta. No se pudo obviar, empero, que existían otros estándares de TESOL que coincidían con al menos otras dos referencias y que debían considerarse como importantes también. Entonces, se aumentó la cantidad de 'mínimos definitivos' a seis.

A los 'mínimos definitivos' se agregaron 3 más: 1.3 los profesores de idioma se esfuerzan para expandir sus habilidades y conocimientos para evaluar, adoptar y adaptar tecnologías emergentes a lo largo de sus carreras (TESOL, 2008, p. 29), 2.2 los profesores de idioma integran coherentemente la tecnología con los enfoques pedagógicos (TESOL, 2008, p. 32), y 3.2 los profesores de idioma utilizan recursos tecnológicos para recolectar y analizar información para mejorar la instrucción y enseñanza del idioma (TESOL, 2008, p. 38).

Una vez seleccionados los estándares, y considerando que serían usados como temas de entrevista y variables operacionales, se decidió a cambiar el nombre. El nombre original era el estándar completo de TESOL. En la práctica, el nombre resulta muy largo. Es decir, los nombres fueron cambiados para una mejor organización y manejo. No obstante, aún cuando el nombre cambió, el estándar y la descripción siguen siendo los mismos.

Para elegir el nombre se decidió analizar el estándar, la definición y los indicadores. Primeramente, se evaluó el estándar mismo. Se analizó el texto del estándar y se contrastó con la definición dada por los mismos autores, Healey y Hubbard (s.f.). Luego, sabiendo que cada indicador es excluyente de otros (Sabino, 1992), se decidió que serían estos, junto con la descripción de cada estándar, los que definirían el nombre final que adoptarían como variable.

De esta manera, las variables fueron llamadas (ver Tabla 1):

Empleo básico: estándar TESOL (2008) 1.1

Material de apoyo: estándar TESOL (2008) 1.2

Actualización y mejoramiento: estándar TESOL (2008) 1.3

Integración a la enseñanza: estándar TESOL (2008) 2.2

Alcance curricular: estándar TESOL (2008) 2.3

Integración a la evaluación: estándar TESOL (2008) 3.2 


\section{Tabla 1}

\begin{tabular}{|c|c|c|}
\hline $\begin{array}{l}\text { Nombre } \\
\text { tema/ } \\
\text { variable }\end{array}$ & Definición del estándar & Indicadores \\
\hline \multirow{3}{*}{$\begin{array}{l}\text { Empleo } \\
\text { básico } \\
\text { (TESOL, } \\
2008,1.1)\end{array}$} & \multirow{3}{*}{$\begin{array}{l}\text { Los profesores de idiomas } \\
\text { deben saber más que sus } \\
\text { estudiantes en materia } \\
\text { de tecnología. También } \\
\text { deben tener conocimientos } \\
\text { generales en materia de } \\
\text { TIC (Healey y Hubbard, } \\
\text { s.f., p. 14). }\end{array}$} & $\begin{array}{l}\text { Llevan a cabo funciones básicas } \\
\text { en dispositivos digitales para } \\
\text { lograr metas instruccionales y } \\
\text { organizacionales }\end{array}$ \\
\hline & & $\begin{array}{l}\text { Preparan material de trabajo } \\
\text { para los estudiantes usando } \\
\text { herramientas digitales } \\
\text { (procesadores de palabra, } \\
\text { presentaciones, recursos en } \\
\text { línea, etc.). }\end{array}$ \\
\hline & & $\begin{array}{l}\text { Tienen los cuidados necesarios } \\
\text { al usar recursos y comunicarse } \\
\text { en línea o electrónicamente. }\end{array}$ \\
\hline \multirow{3}{*}{$\begin{array}{l}\text { Material } \\
\text { de apoyo } \\
\text { (TESOL, } \\
2008,1.2)\end{array}$} & \multirow{3}{*}{$\begin{array}{l}\text { Los profesores deben co- } \\
\text { nocer la tecnología, en ma- } \\
\text { teria de hardware, softwa- } \\
\text { re y red, disponible en su } \\
\text { contexto educativo y saber } \\
\text { cómo usarla correctamente } \\
\text { para mejorar el proceso de } \\
\text { enseñanza (Healey y Hub- } \\
\text { bard, s.f., p. 15). }\end{array}$} & $\begin{array}{l}\text { Comparten información con } \\
\text { colegas sobre la tecnología } \\
\text { disponible. }\end{array}$ \\
\hline & & $\begin{array}{l}\text { Usan tecnología en línea para } \\
\text { dar material de trabajo y de } \\
\text { apoyo. }\end{array}$ \\
\hline & & $\begin{array}{l}\text { Localizan y adaptan una amplia } \\
\text { variedad de material en línea. }\end{array}$ \\
\hline \multirow{4}{*}{$\begin{array}{l}\text { Actualiza- } \\
\text { ción y me- } \\
\text { joramiento }\end{array}$} & \multirow{4}{*}{$\begin{array}{l}\text { Debido a que la tecnología } \\
\text { cambia constantemente, } \\
\text { los profesores de inglés } \\
\text { debe esforzarse para } \\
\text { continuar aprendiendo } \\
\text { de tecnología mientras } \\
\text { se mantenga enseñando } \\
\text { (Healey y Hubbard, s.f., } \\
\text { p. 16). }\end{array}$} & $\begin{array}{l}\text { Utilizan tecnología para mejorar } \\
\text { una actividad convencional. }\end{array}$ \\
\hline & & $\begin{array}{l}\text { Se mantienen informados } \\
\text { mediante distintas fuentes } \\
\text { (libros, journals, convenciones, } \\
\text { etc.). }\end{array}$ \\
\hline & & $\begin{array}{l}\text { Participan en una comunidad de } \\
\text { práctica relevante. }\end{array}$ \\
\hline & & $\begin{array}{l}\text { Exploran las posibilidades } \\
\text { de incorporar tecnologías } \\
\text { emergentes con una mirada } \\
\text { crítica. }\end{array}$ \\
\hline
\end{tabular}




\begin{tabular}{|c|c|c|}
\hline \multirow{4}{*}{$\begin{array}{l}\text { Integra- } \\
\text { ción a la } \\
\text { enseñanza } \\
\text { (TESOL, } \\
\text { 2008, 2.2). }\end{array}$} & \multirow{4}{*}{$\begin{array}{l}\text { La tecnología debe ser } \\
\text { aplicada por los profesores } \\
\text { de manera lógica, a fin } \\
\text { de que brinde el mismo } \\
\text { apoyo que los enfoques } \\
\text { sin tecnología para que } \\
\text { se lleguen a las metas } \\
\text { deseadas (Healey y } \\
\text { Hubbard, s.f., p. } 20 \text { ). }\end{array}$} & $\begin{array}{l}\text { Reconocen su propio enfoque } \\
\text { de enseñanza para luego } \\
\text { integrar la tecnología a él. }\end{array}$ \\
\hline & & $\begin{array}{l}\text { Demuestran que conocen el } \\
\text { potencial y las limitaciones de } \\
\text { la tecnología. }\end{array}$ \\
\hline & & $\begin{array}{l}\text { Se preparan profesionalmente } \\
\text { en el uso de la tecnología. }\end{array}$ \\
\hline & & $\begin{array}{l}\text { Realizan evaluaciones propias } \\
\text { sobre el uso de la tecnología en } \\
\text { clase. }\end{array}$ \\
\hline \multirow{3}{*}{ 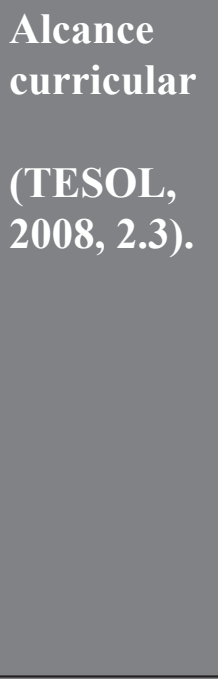 } & \multirow{3}{*}{$\begin{array}{l}\text { Los profesores no deben } \\
\text { seguir al pie de la letra } \\
\text { lo que indica el libro de } \\
\text { texto o el software. Imple- } \\
\text { mentan, adaptan e incluso } \\
\text { crean actividades y tareas } \\
\text { que concuerden con el } \\
\text { currículo. Deben asegurar- } \\
\text { se de que los estudiantes } \\
\text { trabajen como se debe } \\
\text { e intervenir si no es así } \\
\text { (Healey y Hubbard, s.f., } \\
\text { p. 21). }\end{array}$} & $\begin{array}{l}\text { Conocen el nivel de } \\
\text { competencia digital de sus } \\
\text { estudiantes. }\end{array}$ \\
\hline & & $\begin{array}{l}\text { Eligen tecnología que se alinee } \\
\text { a las necesidades y habilidades } \\
\text { de sus estudiantes. }\end{array}$ \\
\hline & & $\begin{array}{l}\text { Se aseguran que los estudiantes } \\
\text { sepan cómo usar la tecnología } \\
\text { para lograr objetivos educativos }\end{array}$ \\
\hline \multirow{4}{*}{$\begin{array}{l}\text { Integra- } \\
\text { ción a la } \\
\text { evaluación } \\
\text { (TESOL, } \\
2008,3.2) \text {. }\end{array}$} & \multirow{4}{*}{$\begin{array}{l}\text { Los profesores utilizan sus } \\
\text { habilidades tecnológicas } \\
\text { para recolectar y analizar } \\
\text { información y datos sobre } \\
\text { materiales de trabajo y el } \\
\text { rendimiento del estudiante } \\
\text { (Healey y Hubbard, s.f., } \\
\text { p. 24). }\end{array}$} & $\begin{array}{l}\text { Demuestran estar familiarizados } \\
\text { con principios relacionados } \\
\text { a evaluación mejorada con } \\
\text { tecnología. }\end{array}$ \\
\hline & & $\begin{array}{l}\text { Usan resultados de evaluaciones } \\
\text { mejoradas con tecnología para } \\
\text { planear sus clases. }\end{array}$ \\
\hline & & $\begin{array}{l}\text { Pueden interpretar resultados de } \\
\text { evaluaciones computarizadas } \\
\text { para los interesados. }\end{array}$ \\
\hline & & $\begin{array}{l}\text { Piden retroalimentación de sus } \\
\text { estudiantes para mejorar el uso } \\
\text { de la tecnología en clase. }\end{array}$ \\
\hline
\end{tabular}


La tabla 1 muestra tres columnas: nombre tema/variable, definición del estándar e indicadores. En la primera columna se muestran seis temas o variables distintas. Estos son los estándares de TESOL (2008) con el cambio de nombre. La segunda columna muestra la definición de Healey y Hubbard (s.f.), que es la definición del estándar de TESOL. Esta es una explicación de lo que busca el estándar. La tercera columna muestra los indicadores de cada estándar. Estos fueron tomados de TESOL (2008), son los mismos indicadores, sin modificar. Se recalca que la información fue traducida del documento original.

Estos son los estándares filtrados que pueden ser considerados para el contexto mexicano. No obstante, deben ser investigados de una forma cualitativa. Los estándares, aún siendo una medida normalizada para conocer las características de un objeto o sujeto (Ravitch, 1995) no deberían ser consideradas únicos, inamovibles, inmodificables o perfectos. Mishler, (1991) y Murphy-Judy y Youngs (2006), entre algunos otros, mencionan que la estandarización de procesos no debería dictar el único camino, sino un posible camino a seguir. De esta forma, en México queda aún la tarea de conocer si los estándares internacionales, bajo los que en ocasiones se siguen las políticas tecnológicas de educación, son apropiados para nuestro contexto. De ser así, podrían tener pautas que guíen la implementación de TIC en la educación en general y en la enseñanza de idiomas, en particular del idioma inglés. En caso de no ser apropiadas, deberán modificarse o crearse a partir de bases ya definidas para que sean adecuadas en el contexto mexicano.

\section{Conclusión}

Para el contexto mexicano, no existen estándares tecnológicos definidos para un profesor de idiomas. Todo lo que existe disponible son documentos internacionales que se aplican en contextos variados, pero que no siempre se consideran los más aptos para contextos o disciplinas específicas. Aún cuando la enseñanza del idioma inglés es de suma importancia en México (por motivos geográficos, culturales y políticos), y que la incursión de las TIC en la educación mexicana se ha ido incrementando, se tiene en cuenta que poco se ha hecho en materia de política o desarrollo curricular.

El contexto mexicano necesita la creación de estándares propios. Este trabajo puede considerarse un paso hacia esa dirección. No 
se intenta que los mínimos aquí mostrados sean considerados únicos, pero pueden considerarse como directrices para guiar una investigación mayor que permita que se consideren como los mínimos o los máximos, dependiendo de una investigación a profundidad y cualitativa que se realice en instituciones educativas propias del contexto mexicano. Incluso sería apropiada la modificación de los estándares mínimos aquí propuestos, siempre considerando que pueden ser modificados y revisados a futuro para tener una mayor calidad en la enseñanza de inglés apoyada con tecnología.

\section{Referencias bibliográficas}

Alarcón, E. y Ortiz, V. (2014). Estudiantes, profesores y TIC. La investigación en México. En A. Ramírez y M.A., Casillas Háblame de TIC (pp. 39-70). Córdoba, Argentina: Editorial Brujas.

Ander-Egg, E. (1995) Técnicas de investigación social. Buenos Aires, Argentina: Editorial Lumen.

Brünner, J.J. (2003). Educación e internet. ¿La próxima revolución? Chile: Fondo de Cultura Económica.

Cambridge English. (2014a). Cambridge English Teaching framework - How and why the Teaching Framework was developed. Recuperado de http://www.cambridgeenglish.org/teaching-english/ cambridge-english-teaching-framework/

Cambridge English. (2014b). Cambridge English Teaching framework - Categories and components. Recuperado de http://www.cambridgeenglish.org/teaching-english/ cambridge-english-teaching-framework/

Cambridge English. (2014c). Cambridge English Teaching frameworkFull level descriptors. Recuperado de http://www.cambridgeenglish.org/teaching-english/cambridge-english-teaching-framework/

Carney, N. y Foss, P. (2008). Student-produced video: two approaches. English Teaching Forum, 46 (2), pp. 14-19.

Castells, M. (1999). La era de la información: economía, sociedad y cultura. Volumen I: La sociedad red. México, D.F: Siglo Veintiuno.

Chapelle, C.A. (2001). Computers applications in second language acquisition. Foundations for teaching, testing and research. Cambridge, UK: Cambridge University Press. 
Clark, B. (1983). El sistema de educación superior. Una visión comparativa de la organización académica. México: Nueva Imagen.

Crovi, D. (2009) Acceso, uso y apropiación de las TIC en comunidades académicas. 1ra Ed. México, D.F.: UNAM

Garrett, N. (2009) Computer-Assisted Language Learning Trends and Issues Revisited: Integrating Innovation. The Modern Language Journal, 93, pp. 719-740.

Garza, A. (2004). Manual de técnicas de investigación para estudiantes de ciencias sociales. México: El Colegio de México.

Gordo, A. y Serrano, A. (2008). Estrategias y prácticas cualitativas de investigación social. Madrid, España: Pearson Educación.

Gruba, P. y Hinkelman, D. (2012). Blending technologies in second language classrooms. New York, NY: Palgrave Macmillan.

Harmer, J. (2001). The practice of English language teaching. Malasia: Pearson Education Limited.

Healey, D. y Hubbard, P. (s.f.) Introducing the New TESOL Technology Standards.

Keats, D. (1992). La entrevista perfecta. México, D.F.: Editorial Pax México.

Mishler, E.G. (1991). Representing discourse: the rethoric of transcription. Journal of narrative and life history, 1 (4), pp. 255-280.

Murphy-Judy, K. y Youngs, B.L. (2006). Technology standards for teacher education, credentialing and certification. En M. Levy y P. Hubbard (Eds.), Teacher education in CALL (pp. 45-60). Amsterdam, Holanda: John Benjamins Publishing Company.

National Council of Teacher of English NCTE. (1996). Standards for the English Language arts. Recuperado de http://www.ncte.org/ standards/ncte-ira

O’Donoghue, M. (2014) Producing video for teaching and learning: a framework for planning and collaboration. Nueva York, EUA: Routledge.

Piper, A. (1987). Helping learners to write: a role for the word processor. ELT Journal, 41 (2), pp. 119-125.

Ramírez-Martinell, A., Casillas, M.A. y Contreras, C.C., (2014). La incorporación de las TIC a la enseñanza universitaria de los idiomas. Debate Universitario, 5 (noviembre 2014), pp. 123-138.

Ravitch, D. (1995). National standards in American education: a citizen's guide. Washington, D.C: Brookings 
Richards, J.C. (2008). Growing up with TESOL. English language forum, 46 (1), pp. 2-11.

Richards, J.C. (2011). Competence and performance in language teaching. Nueva York, EUA: Cambridge University Press.

Richards, J.C. y Farrell, T.S.C. (2005). Professional development for language teachers. Nueva York, EUA: Cambridge University Press

Rodarte, R. (2014). Las tecnologías de la información y la comunicación en la educación musical: Compilaciones en el aula. En A. Ramírez y M.A. Casillas Háblame de TIC (pp. 135-156). Córdoba, Argentina: Editorial Brujas.

Sabino, C. (1992). El proceso de investigación. Caracas, Venezuela: Panapo. Salsbury, T. y Crummer, C. (2008). Using teacher-developed corpora in the CBI classroom. English Teaching Forum, 46 (2), pp. 28-37.

SEP (2011a) Programa Nacional de Inglés en Educación Básica PNIEB - Fundamentos curriculares - Fase de expansión

SEP (2011b) Programa Nacional de Inglés en Educación Básica PNIEB - Programas de estudio 2011 Ciclo 4

SEP (s.f.) Perfiles de docentes y asesores técnico-pedagógicos para la asignatura de inglés en educacioón baásica

TESOL. (2008). TESOL Technology Standards Framework. Retrieved from http://www.tesol.org/docs/books/bk_technologystandards_ framework_721.pdf

Toffler, A. (1980). La tercera ola. Barcelona, España: Plaza y Janés Editores. Universidad de Southampton. (s.f.) European profile for language teacher education - A frame of reference.

Wells, S. (2010). Technology, genre, and gender. The case of power structure research. En S.A. Selber (Ed.), Rethorics and technologies. New directions in writing and communications. Columbia, EUA: University of South Carolina Press. 